\section{Läufer-Knie erfolgreich mit BoNT behandeln}

\author{
Neben der zugelassenen Anwendung von BoNT-A zur Behandlung der \\ Spastik der Beinmuskulatur nach Schlaganfall oder bei infantiler Zerebral- \\ parese wäre auch ein Einsatz in der Sportmedizin denkbar.
}

$Z_{s}$ den arzneimittelrechtlich zugelassenen Anwendungen von Botulinumneurotoxin A (BoNT-A) gehört die Behandlung der Spastik der Beinmuskulatur infolge eines Schlaganfalls oder infantiler Zerebralparese. Die intramuskuläre Injektion von BoNT-A wird gelegentlich auch als Off-Label-Therapie zur gezielten Schwächung hypertrophierter Muskeln bei Engpasssyndromen (z.B. $M$. scalenus anterior bei neurogenem Thoracic-Outlet-Syndrom, M. piriformis bei Piriformis-Syndrom) eingesetzt. In der Sportmedizin spielt die Injektionsbehandlung mit BoNT-A bislang eine untergeordnete Rolle. Einige seit 2006 publizierte Pilotstudien, davon eine kleine randomisierte Studie, sprechen für einen günstigen Effekt der einmaligen EMG-gesteuerten BoNT-A-Injektion in den M. vastus lateralis beim chronischen, therapierefraktären patellofemoralen Schmerzsyndrom (PFSS).

Das PFSS (Synonym: Chondropathia patellae, parapatellares Schmerzsyndrom, Chondromalacia patellae) ist ein uneinheitliches Krankheitsbild, das durch eine Über- oder Fehlbelastung der proximalen Beinmuskulatur ausgelöst wird. Neben einer entzündlichen Reizung des Tractus iliotibialis (iliotibiales Bandsyndrom [ITBS], Synonym: LäuferKnie, Tractus-Syndrom) kann ursächlich auch eine Bursitis oder Tendinitis dem PFSS zugrunde liegen. Daher waren Patienten mit Bursitis, Tendinitis, hypermobiler Patella, früherer Patelladislokation oder deutlichem Übergewicht $(\mathrm{BMI}>30)$ von diesen Pilotstudien ausgeschlossen worden, so dass nur Patienten mit einem chronischen ITBS mittels BoNT-A behandelt wurden. Bislang ist unklar, ob die BoNT-A-Injektion in den $M$. vastus lateralis oder in den M. tensor fasciae latae einen besseren Therapieerfolg erbringt. Zudem liegen bislang kaum Daten über die Langzeitwirkung dieser Behandlung vor.

Die hier vorgestellte Studie untersucht erstmals über einen Zeitraum von fünf
Jahren in einer einarmigen prospektiven Studie die Effektivität der ultraschallgezielten BoNT-A-Injektion in den M. tensor fasciae latae bei Patienten mit ITBS. Eingeschlossen wurden 45 erwachsene Patienten mit einem aktivitätsassoziierten ITBS mit einer Symptomdauer von mindestens drei Monaten. Die Diagnose des ITBS musste mittels MRT des Oberschenkels belegt worden sein. Der Knieschmerz musste durch Anspannung des iliotibialen Traktes im modifizierten Ober-Test reproduzierbar auslösbar sein, und eine mindestens sechswöchige Physiotherapie musste erfolglos geblieben sein. Ausschlusskriterien waren eine Subluxation oder Dislokation der Patella, eine Tendinopathie der Patella und eine ausgeprägte patellofemorale Osteoarthritis.

Alle Patienten erhielten eine ultraschallgezielte Injektion von 75 Einheiten BoNT-A (Abobotulinumtoxin A, Dysport ${ }^{\circ}$; Ipsen) in $0,75 \mathrm{ml}$ isotonischer Kochsalzlösung in den zentralen Muskelbauch des M. tensor fasciae latae, ausgeführt von einem Radiologen mit Erfahrung in muskuloskelettaler Bildgebung. Anschließend erfolgten standardisierte Untersuchungen und Behandlungen durch spezialisierte Physiotherapeuten. Alle Patienten wurden vor der Behandlung sowie eine, vier und zwölf Wochen und fünf Jahre danach durch einen erfahrenen Untersucher beurteilt. Das primäre Outcome-Kriterium war die Selbstbeurteilung des Knieschmerzes anhand der "Anterior Knee Pain Scale“(AKPS; Kujala-Score). Dabei handelt es sich um einen Fragebogen mit 13 kniebezogenen Fragen und einem maximalen Score von 100, wobei höhere Scores geringere Beschwerden bedeuten. Das sekundäre Outcome-Kriterium war die Länge des Tractus iliotibialis, bestimmt mit dem modifizierten OberTest. 39 der 45 Patienten konnten nach fünf Jahren nachuntersucht werden.

Als wesentliches Ergebnis zeigte sich eine über fünf Jahre anhaltende Besse-

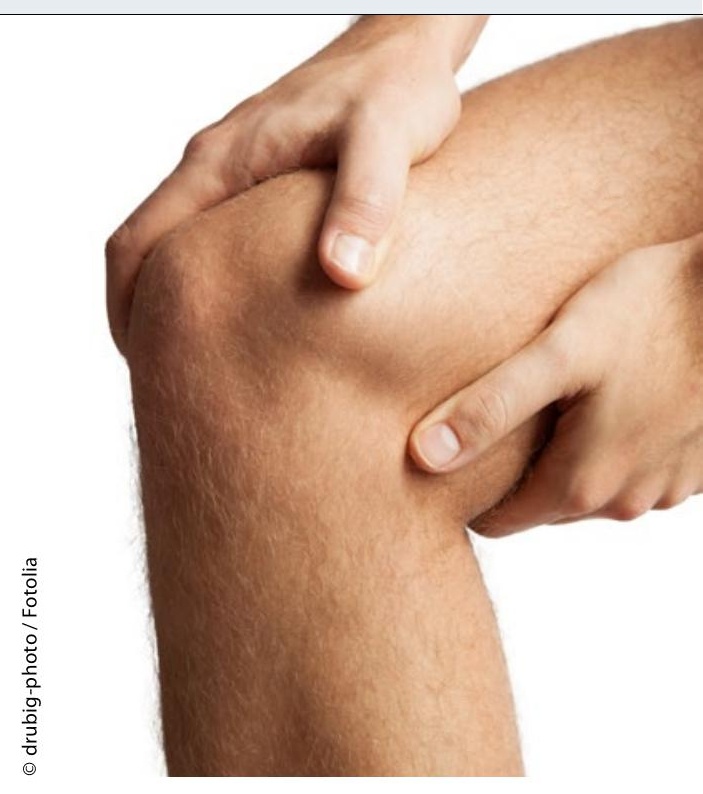

rung des Knieschmerzes, gemessen mittels der AKPS, mit einem mittleren initialen Score von 61 und einem Score von 87 nach fünf Jahren. Zudem zeigte sich eine signifikante Verlängerung des iliotibialen Traktes. Mittels Computersimulation wurde eine Aktivitätsnormalisierung des $M$. gluteus medius infolge der dosierten Schwächung des M. tensor fasciae latae ermittelt.

Vier Patienten erhielten ein oder zwei Re-Injektionsbehandlungen, die dann zu einem Sistieren der Kniebeschwerden führten. Lediglich bei zwei Patienten waren nur Kurzzeiteffekte zu erreichen, so dass diese schließlich operativ behandelt wurden. $69 \%$ der Patienten wurden völlig schmerzfrei. Ebenso $69 \%$ der Patienten konnten ihre sportliche Aktivität wieder auf dem früheren Niveau fortführen.

\section{Kommentar}

Zusammenfassend wird hier erstmals in einer prospektiven Langzeitstudie gezeigt, dass die ultraschallgezielte BoNT-A-Injektion in den M. tensor fasciae latae einen anhaltenden Behandlungserfolg bei erwachsenen Patienten mit therapieresistentem ITBS erbringt. Allerdings ist die Replikation dieser Ergebnisse in weiteren, möglichst randomisierten Studien erforderlich, bevor eine breite Anwendung in dieser Indikation empfohlen werden kann.

\section{Prof. Dr. med. Uwe Walter}

Stephen JM et al. The use of sonographically guided botulinum toxin type A (Dysport) injections into the tensor fasciae latae for the treatment of lateral patellofemoral overload syndrome. Am J Sports Med. 2016; 44 (5): 1195-1202 